\title{
BMJ Global Health Improving maternal, newborn and child health outcomes through a community- based women's health education program: a cluster randomised controlled trial in western Kenya
}

\author{
Lauren Y Maldonado (D) , ${ }^{1,2}$ Jeffrey Bone (D) , ${ }^{3}$ Michael L Scanlon, ${ }^{2,4}$ \\ Gertrude Anusu, ${ }^{2}$ Sheilah Chelagat, ${ }^{2}$ Anjellah Jumah, ${ }^{2}$ Justus E Ikemeri, ${ }^{2}$ \\ Julia J Songok, ${ }^{2,5}$ Astrid Christoffersen-Deb, ${ }^{3,6}$ Laura J Ruhl ${ }^{2,7}$
}

To cite: Maldonado LY, Bone J, Scanlon ML, et al. Improving maternal, newborn and child health outcomes through a community-based women's health education program: a cluster randomised controlled trial in western Kenya. BMJ Global Health 2020;5:e003370. doi:10.1136/ bmjgh-2020-003370

Handling editor Seye Abimbola

- Additional material is published online only. To view please visit the journal online (http://dx.doi.org/10.1136/ bmjgh-2020-003370).

JJS, AC-D and LJR contributed equally.

Received 6 July 2020

Revised 17 October 2020

Accepted 20 0ctober 2020

Check for updates

C Author(s) (or their employer(s)) 2020. Re-use permitted under CC BY-NC. No commercial re-use. See rights and permissions. Published by BMJ.

For numbered affiliations see end of article.

Correspondence to Dr Lauren Y Maldonado; lymaldonado@mgh.harvard.edu

\section{ABSTRACT}

Introduction Community-based women's health education groups may improve maternal, newborn and child health (MNCH); however, evidence from subSaharan Africa is lacking. Chamas for Change (Chamas) is a community health volunteer (CHV)-led, groupbased health education programme for pregnant and postpartum women in western Kenya. We evaluated Chamas' effect on facility-based deliveries and other MNCH outcomes.

Methods We conducted a cluster randomised controlled trial involving 74 community health units in Trans Nzoia County. We included pregnant women who presented to health facilities for their first antenatal care visits by 32 weeks gestation. We randomised clusters 1:1 without stratification or matching; we masked data collectors, investigators and analysts to allocation. Intervention clusters were invited to bimonthly, group-based, CHV-led health lessons (Chamas); control clusters had monthly, individual CHV home visits (standard of care). The primary outcome was facility-based delivery at 12-month followup. We conducted an intention-to-treat approach with multilevel logistic regression models using individual-level data.

Results Between 27 November 2017 and 8 March 2018, we enrolled 1920 participants from 37 intervention and 37 control clusters. A total of $1550(80.7 \%)$ participants completed the study with $822(82.5 \%)$ and $728(78.8 \%)$ in the intervention and control arms, respectively. Facilitybased deliveries improved in the intervention arm $(80.9 \%$ vs $73.0 \%$; risk difference (RD) $7.4 \%, 95 \% \mathrm{Cl} 3.0$ to $12.5, \mathrm{OR}=1.58,95 \% \mathrm{Cl} 0.97$ to $2.55, \mathrm{p}=0.057)$. Chamas participants also demonstrated higher rates of 48 hours postpartum visits (RD 15.3\%, 95\% Cl 12.0 to 19.6), exclusive breastfeeding (RD $11.9 \%, 95 \% \mathrm{Cl} 7.2$ to 16.9 ), contraceptive adoption (RD 7.2\%, 95\% Cl 2.6 to 12.9 ) and infant immunisation completion (RD 15.6\%, 95\% Cl 11.5 to 20.9).

Conclusion Chamas participation was associated with significantly improved MNCH outcomes compared with the standard of care. This trial contributes robust data from sub-Saharan Africa to support community-based, women's

\section{Key questions}

What is already known?

- Globally, maternal and infant deaths have declined over the last three decades; however, low and middle-income countries (LMICs) disproportionately incur the highest morbidity and mortality.

- The WHO recommends leveraging lay health workers (LHWs), including community health volunteers (CHVs), to promote maternal, newborn and child health (MNCH) in resource-limited settings.

- Prior research suggests coupling community-based approaches (ie, LHW-led interventions) and women's health education groups during pregnancy and postpartum may improve MNCH outcomes; however, robust evidence from sub-Saharan Africa is lacking.

What are the new findings?

- Using a cluster randomised controlled trial design, we found participation in Chamas for Change (Chamas) - a group-based women's health education programme led by CHVs-was associated with significantly improved rates of facility-based deliveries compared with the standard of care (ie, individual, monthly home visits) in rural Kenya.

- This trial also demonstrated significant associations between programme participation and receiving 48 hour postpartum home visits, breastfeeding exclusively, adopting a contraceptive method postpartum and immunising infants fully by 12 months of life compared with the standard of care.

- These findings support pilot data from a preceding evaluation of the Chamas programme as well as the current literature on community-based MNCH interventions led by LHWs in other LMICs.

health education groups for MNCH in resource-limited settings.

Trial registration number

NCT03187873. 


\section{Key questions}

What do the new findings imply?

- Effective community-based strategies that promote MNCH are needed to continue to improve the health and well-being of women and infants in rural sub-Saharan Africa and other LMICs.

- Chamas offers an innovative approach that leverages existing community infrastructure to improve MNCH in a rural, resource-limited setting with significant health policy implications.

- Collective evidence from this trial and preceding studies support community-based women's health education groups as an effective strategy for improving uptake of facility-based deliveries and other life-saving MNCH practices.

\section{INTRODUCTION}

Globally, maternal and infant deaths have declined over the last three decades; however, low and middleincome countries (LMICs) still disproportionately incur the highest morbidity and mortality. Kenya's maternal mortality ratio (MMR) and infant mortality rate (IMR) remain among the highest in the world at 342 per 100000 live births and 31 per 1000 live births, respectively. ${ }^{12}$ Fragile health systems, poor access to high quality and specialised care, low health literacy rates, gender-based inequities and generational poverty contribute to this disparity. ${ }^{3-5}$ Effective solutions that build on infrastructure to promote the health and well-being of women and infants are needed to continue to improve maternal, newborn and child health $(\mathrm{MNCH})$ outcomes in resource-limited settings.

Mobilising community health volunteers (CHVs) to promote $\mathrm{MNCH}$ offers a promising strategy to reduce health inequities. ${ }^{6-8}$ In 2006, the Republic of Kenya Ministry of Health (MOH)'s 'Kenya Essential Package for Health' delineated a comprehensive strategy to improve the health of households and communities, commonly known as the 'Community Health Strategy' (CHS). ${ }^{9}$ Under the current CHS, CHVs are expected to perform monthly, individual home visits for all pregnant women during pregnancy and throughout the first year postpartum. ${ }^{10}$ Despite these efforts, the practice of $\mathrm{MNCH}$ interventions associated with reductions in mortality and morbidity (ie, facility-based deliveries with skilled birth attendants) are well-below projected targets to substantively reduce the MMR and IMR. ${ }^{1}$ These gaps are pronounced across socioeconomic and geographic strata with women in poorer, rural communities experiencing significantly worse outcomes than those in wealthier, urban centres.

The WHO recommends integrating lay health workers, including $\mathrm{CHVs}$, to promote $\mathrm{MNCH}$ interventions. ${ }^{11}$ Coupling this strategy with the delivery of group-based women's health education may improve $\mathrm{MNCH}$; however, evidence from sub-Saharan Africa is limited. ${ }^{12}$ Aggregate data from cluster-randomised and quasi-randomised trials from Nepal, India, Bangladesh and Malawi underscore the value of community-centred, group-based health promotion to improve maternal and newborn care. ${ }^{13}$ Though causal mechanisms to explain the benefit of group-based interventions remain speculative, fostering mechanisms for peer accountability and support may play a significant role. ${ }^{14}$

In 2012, the Academic Model Providing Access to Healthcare (AMPATH) - a long-standing partnership between the Kenyan MOH, Moi University, Moi Teaching and Referral Hospital and North American universities-launched Chamas for Change (Chamas). This programme leveraged the success of existing groupbased health education models in an effort to improve $\mathrm{MNCH}$ in Kenya as well as generate evidence to support these interventions in sub-Saharan Africa. Chamas is a CHV-led, group-based health education programme that supports women during the first 1000 days of life (ie, pregnancy, infancy and toddlerhood). The programme hybridises best practices from resource-limited settings globally to offer a community-based, multipronged strategy for improving MNCH. This strategy focuses on providing health education, a peer-supportive environment and opportunities to access financial capital to promote $\mathrm{MNCH}$ while simultaneously addressing inequities that perpetuate poor outcomes.

A pilot study investigating first-year Chamas participation demonstrated significant associations between participation and the likelihood of practising positive $\mathrm{MNCH}$ behaviours such as delivering in a health facility with a skilled birth attendant. ${ }^{15}$ To validate whether first-year Chamas participation is positively associated with health facility delivery and the practice of other key MNCH interventions, we conducted a large-scale cluster randomised controlled trial in rural western Kenya. We hypothesised women participating in Chamas would be more likely to deliver in health facilities than those receiving individual, home-based visits (standard of care).

\section{METHODS \\ Study design}

We conducted a two-arm cluster randomised controlled trial in 74 community health units (CUs) across four subcounties (Cherangany, Kwanza, Kiminini and Saboti) in Trans Nzoia County, Western Province, Kenya (figure 1). Figure 1 depicts these 74 CUs allocated to control and intervention trial arms. Cluster randomisation was used to avoid potential contamination of intervention activities between neighbouring villages. Additional study details are available in our trial protocol (online supplemental trial protocol). We obtained written informed consent from all participants prior to data collection. We adhered to Consolidated Standards of Reporting Trials guidelines for reporting results of cluster randomised controlled trials (online supplemental CONSORT checklist).

\section{Participants}

We identified 77 CUs among 163 total CUs across our four selected subcounties in Trans Nzoia to serve as 


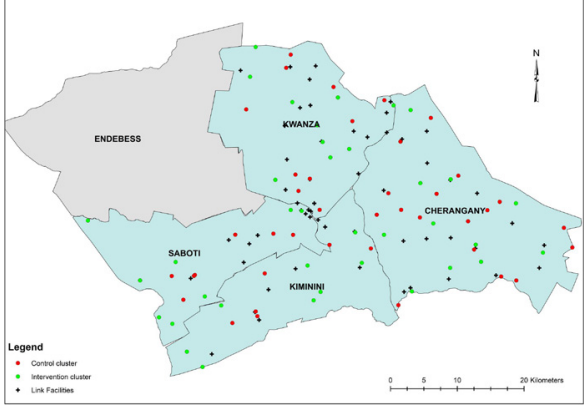

Figure 1 Cluster map.

potential clusters. CUs are geographically defined health service delivery areas, $5-8 \mathrm{~km}^{2}$ in size, for populations of 5000 people supervised by Community Health Extension Workers (CHEWs) and CHVs. CHEWs and CHVs connect CUs with their assigned health facilities (or 'link facilities'), extending services traditionally based at facilities to the household level. CHVs are nominated members of their communities who serve as liaisons between community members and the health sector. CHEWs are salaried frontline health workers responsible for supervising CHVs. CUs selected for this trial were specifically chosen as their $\mathrm{CHVs}$ received formal CHS training from AMPATH.

We recruited participants from 60 public and private health facilities linked to our 77 identified CUs. Pregnant women who were less than or equal to 32 weeks gestation, presenting for their first antenatal care (ANC) visits and residing in one of the 77 CUs were eligible. Among 77 identified CUs, 74 were represented by women deemed eligible for participation. We selected a gestational age cut-off of 32 weeks as the majority (96.0\%) of Kenyan women who seek ANC at any point during pregnancy present for at least one $\mathrm{ANC}$ visit by this time. ${ }^{1}$ Due to slow recruitment resultant of preceding health worker strikes in Trans Nzoia, we increased our original gestational cut-off from 28 to 32 weeks.

\section{Randomisation and masking}

We randomised CUs selected to serve as clusters 1:1 to intervention (eg, Chamas programme) or standard of care (eg, monthly CHV home visits). The trial data manager used a simple random allocation sequence generated by PASS V.11.0.10) to designate cluster assignment. Nonstudy CUs (ie, those not randomised in this trial) served as buffer zones between intervention and control clusters to avoid contamination. There was no stratification or matching. We masked data collectors (trained AMPATH research assistants), investigators and analysts to cluster allocation throughout the trial; however, both arms were identifiable to participants and CHVs by design.

\section{Procedures}

Data collectors assessed women for eligibility at their first ANC visit. Women deemed eligible and willing to participate provided consent to be contacted for enrolment. The data manager generated lists of participants organised by residential CUs. These lists were subsequently distributed to CHVs who were tasked with finding women in their respective CUs and enrolling them. Data collectors accompanied CHVs during this process and obtained baseline data at enrolment. One week following the end of the enrolment period, the data manager randomised all CUs to intervention and control arms. Three weeks later, CHVs began facilitating Chamas in intervention clusters.

Intervention clusters participated in the Chamas programme (programme details are published elsewhere). ${ }^{15}$ Briefly, Chamas is a group-based, CHV-led health education programme that supports women during the first 1000 days of their child's life. Women randomised to the intervention arm participated in Chamas in lieu of receiving individual home visits (standard of care). Participants attend 60-90 min sessions two times a month, which include discussions on health and social topics relevant to antenatal, postpartum and early childhood experiences. CHVs use an illustrated flip chart with evidence-based, structured discussion guides to facilitate lessons. Groups are typically comprised of 15-20 women, two $\mathrm{CHV}$ facilitators and two mentor mothers (eg, postmenopausal women who have completed child rearing). The first year of the curriculum promotes behaviours associated with demonstrated reductions in maternal and infant morbidity and mortality. These lessons purposefully mirror health topics that CHVs are expected to promote during home visits under the CHS. Following each lesson, women are invited to participate in an optional table-banking programme called Group Integrated Savings for Health and Empowerment (GISHE). GISHE participation is optional so as not to deter women without financial means to contribute to group savings from joining Chamas. Women are encouraged to use savings generated by GISHE to finance health interventions (eg, enrol in health insurance, pay for transportation to health facilities), invest in early childhood education and/or start small businesses.

Strategies to ensure fidelity of Chamas included: using standardised intervention materials (ie, printed curriculum flipcharts), hosting structured CHV training sessions preceding the trial, offering monthly supervision by study staff and designating at least two trained CHVs to every group to avoid potential disruptions due to illnesses or job transfers. In addition to attending the 4-day MNCH refresher training, CHVs facilitating Chamas also received a formal 2-day orientation to the programme and were trained in group facilitation techniques. We provided scheduled support sessions for CHV facilitators throughout the trial (during months 1-3, 6, 9 and 12), which provided opportunities for feedback and communal troubleshooting to enhance programme delivery.

Control clusters had monthly CHV home visits during pregnancy and postpartum, as recommended by the Kenyan CHS standard of care. ${ }^{10}$ During monthly visits, $\mathrm{CHVs}$ collect basic health information, identify antenatal 
and early postpartum danger signs, refer individuals to care and aid in infant growth monitoring. CHVs are also expected to encourage women to adopt the same key health behaviours promoted in Chamas. CHVs working within control clusters received oversight and supervision from CHEWs, as structured by the CHS. CHVs performing door-to-door visits typically oversee a catchment of 15 women who are each visited for 20-30 min on a monthly basis (up to 7.5 hours per month). Those facilitating Chamas substituted door-to-door visits with group sessions; as such, their volunteer effort was reduced to two 60-90 min sessions per month (up to 3 hours per month).

We did not provide incentives (monetary or other) for participation to CHVs, CHEWs or participants in either study arm at any point during the trial. CHEWs continued to receive salaries from the $\mathrm{MOH}$ and CHVs, who volunteer in addition to participating in other jobs (ie, as teachers, farmers, labourers), continued to work throughout the trial. Notably, CHVs under the current CHS are not financially compensated for performing door-to-door visits. To reduce potential for confounding, we similarly did not compensate CHVs for facilitating Chamas meetings. We did, however, reimburse all CHVs and CHEWs for travel to meetings and trainings as well as for air-time used to contact participants during recruitment.

\section{Outcomes}

We measured outcomes at the individual level. We selected facility-based delivery as our primary outcome because of the significant association between institutional delivery and reductions in maternal and infant morbidity and mortality. ${ }^{16-18}$ Secondary outcomes included: attending adequate ANC (defined as attending at least four visits per Republic of Kenya MOH guidelines), receiving a 48-hour postpartum home visit, exclusively breastfeeding for 6 months, adopting a modern contraceptive method, immunising infants with the oral polio vaccine within 2 weeks postpartum, immunising infants with the measles vaccine (measles I) by 12 months of age and completing the infant immunisation series per WHO and Republic of Kenya MOH standards by 12 months of age. ${ }^{19-21}$ We additionally collected detailed microfinance data as well as validated questionnaire data on perceived levels of peer support and financial empowerment, which we plan to report in future articles.

Data collectors travelled to participant homes to collect end-line data 12 months following the initiation of Chamas sessions and home visits. Outcome measures were self-reported with the exception of infant immunisations, which were extracted from standard $\mathrm{MOH}$ Maternal Child Health Booklets kept by mothers. All data were recorded using electronic, standardised questionnaires. We classified participants as lost to follow-up after we made three attempts to establish contact over a 2-week period. We conducted abbreviated phone surveys if participants relocated outside of Trans Nzoia County; these abbreviated questionnaires omitted questions on infant immunisations.

At enrolment, we collected baseline participant sociodemographic (age, marital status, maternal education, occupation, poverty probability index scores, insurance status) and reproductive health (previous pregnancy and related outcomes) data. We used the Kenya 2015 Poverty Probability Index (PPI) questionnaire and national poverty line scorecard to estimate participants' poverty likelihood at baseline. ${ }^{22}$ We recorded attendance at each Chamas session to track individual programme participation. A Data and Safety Monitoring Board recorded and investigated adverse events including CHV-reported participant mortalities as well as the cause of death (if known).

\section{Statistical analysis}

We estimated sample size using methods described by Rutterford $e t$ al for a proposed mixed effects regression analysis ${ }^{23}$ using derived baseline estimates. ${ }^{15}$ Assuming a mean cluster size of 20 individuals, 77 clusters (equally allocated between arms), intracluster correlation coefficient (ICC) of 0.44 (based on pilot data) $)^{15}$ and $20 \%$ attrition, we calculated that a total of 1280 individuals would be needed to detect a $4.7 \%$ risk difference (RD) (difference in the rate of facility-based birth at the county vs national-level ${ }^{1}$ with $80 \%$ power at a (two-tailed) significance level of 0.05 ). To determine our recruitment timeline, we assumed $6.3 \%$ of all women of reproductive age would be pregnant at any given time (or roughly 50 women per CU annually). ${ }^{1}$ We determined an enrolment period of roughly 3-4 months adequate to recruit our estimated sample size.

Our primary analyses were intention-to-treat (ITT) and included all participants from randomised clusters who provided baseline and 12-month follow-up data, regardless of the level of participation in Chamas. We summarised all demographic and reproductive health history information between arms with means and SDs as well as medians and IQRs for continuous variables and counts and percentages for categorical variables. We analysed the primary outcome with multilevel logistic regression with a random intercept for cluster, and effects are presented as RDs with $95 \%$ bootstrap CIs and ORs with 95\% Wald-type confidence intervals and $\mathrm{p}$ values. We also report the ICC. We analysed secondary outcomes similarly.

For both primary and secondary outcomes, we conducted several sensitivity analyses. First, to assess the impact of missing outcomes due to loss to follow-up, we used multiple imputation with 10 data sets with the 'jomo' algorithm to account for the multilevel structure of the data; results were then combined using Rubin's rules. ${ }^{24}{ }^{25}$ Second, to assess the possible impact of differences in factors known to be associated with care-seeking behaviours between arms, we adjusted our primary models for PPI score, marital status, null parity and health insurance at time of delivery. A third sensitivity 
analyses combined adjustment and imputation. Finally, we performed a sensitivity analysis restricting our intervention sample to women who attended at least one Chamas session during the trial period.

We assessed the effect of Chamas participation on infant vaccination outcomes similarly, but given the large amount of missing data, no sensitivity analyses with imputation were conducted. Adjusted models for vaccination included maternal education, PPI and insurance at delivery as previous studies demonstrate strong associations between these sociodemographic factors and immunisation adherence. ${ }^{26}$ Further, since vaccination data were missing in approximately $40 \%$ of the sample, we were concerned about selection bias in those reporting the outcome. To account for this, we carried out an additional sensitivity analysis to indicate the amount of unmeasured confounding between trial arm and vaccination that would be needed to explain away the observed differences. ${ }^{27}$

There were no interim analyses. We developed, finalised and signed a statistical analysis plan prior to beginning data analysis [online supplemental statistical analysis plan]. Statistical significance was set at 0.05 and all analyses were conducted using $\mathrm{R}$ statistical software $\left(\right.$ V.3.5.3) ${ }^{28}$

\section{Patient and public involvement}

We sought and incorporated feedback from a multidisciplinary study advisory committee including direct beneficiaries (ie, participating women, CHVs) and key stakeholders (ie, local community leaders, Kenyan MOH representatives) in the initial design and conception of this trial. We designed our questionnaires, data instruments and intervention activities based on qualitative feedback provided by programme participants during Chamas pilot studies. These qualitative questionnaires captured participant perceptions of the strengths and weaknesses of the programme as well as priority areas for continued improvement. Prior to initiating trial activities, we invited CHVs, CHEWs, health facility managers, subcounty $\mathrm{MOH}$ representatives and community leaders to stakeholder meetings to explain the study's purpose and procedures as well as to facilitate understanding of our trial objectives among leadership at the county, subcounty and community levels. Following these meetings, we asked community leaders for permission to begin enrolling participants. All CHVs who agreed to participate also attended a 4-day refresher training on their roles and expectations in promoting MNCH under the Kenyan CHS. We discussed the trial's risks and benefits with all participants before enrolment, including demands on individual time due to programme participation and data collection. We obtained written informed consent from all participants prior to data collection. At the trial's conclusion, we verbally disseminated our preliminary findings to the programme's direct beneficiaries and key stakeholders. We plan to additionally distribute printed summaries of key findings following the trial's publication.

\section{Role of the funding source}

The funders had no role in the research design, collection, analysis or interpretation of data, writing this report or the decision to submit this manuscript for publication. The corresponding author had full access to all data in the study as well as final responsibility for the decision to submit this manuscript for publication.

\section{RESULTS}

Details of our enrolment and inclusion procedures are summarised in figure 2. Between 27 November 2017 and 8 March 2018, we assessed 4235 women for eligibility; 2923 women from 74 clusters met criteria and agreed to be contacted. Three of the original 77 identified clusters did not have eligible participants. CHVs successfully contacted and enrolled 1920 eligible women from 74 community clusters (996 participants in 37 intervention and 924 in 37 control clusters). We collected follow-up data on all clusters between 7 April 2019and 3 July 2019. A total of $1550(80.7 \%)$ participants completed the study at 12-month follow-up: we included 822 in the intervention $(82.5 \%)$ and 728 in the control $(78.8 \%)$ arms for analysis. Among 822 intervention participants who completed the study, $599(72.9 \%)$ attended at least one Chamas session. Among those who attended, mean attendance was 12 (SD 7.8) of 24 total sessions and $48.9 \%$ participated in GISHE. Among controls, the mean number of CHV home visits received was 9 (SD 2.3) of 12 total visits. Participants lost to follow-up were similar in number across study groups and attrition was not significantly associated with sociodemographic or reproductive health characteristics (online supplemental table S2). Notably, those lost to follow-up tended to have lower PPI scores than women who completed the trial.

Participants who completed the study $(\mathrm{n}=1550)$ were similar in baseline characteristics (table 1). Most participants were married, unemployed, completed primary school, possessed health insurance at the time of delivery and carried a previous pregnancy. The median gestational age at enrolment was 22 weeks (IQR 17, 25). The mean PPI score for our study population was 55.13 (SD 20.11); PPI scores differed across study arms with higher values among control compared with intervention participants at baseline. Cluster-level demographics were also well balanced. Across all clusters, CHVs possessed a mean 11.69 (SD 6.32) years of experience. Finally, in the intervention arm, we noted geographic differences among women who attended Chamas and those who never attended (online supplemental table S3).

Primary and secondary outcomes are summarised in table 2 . The overall proportion of health facility delivery was higher among intervention $(80.9 \%, 653$ participants) than control participants $(73.0 \%, 514$ participants). Among women who did not deliver in a health facility $(n=383)$, the most commonly cited reasons across cohorts included: preference to deliver at home or with a traditional birth attendant $(32.1 \%)$, structural challenges 


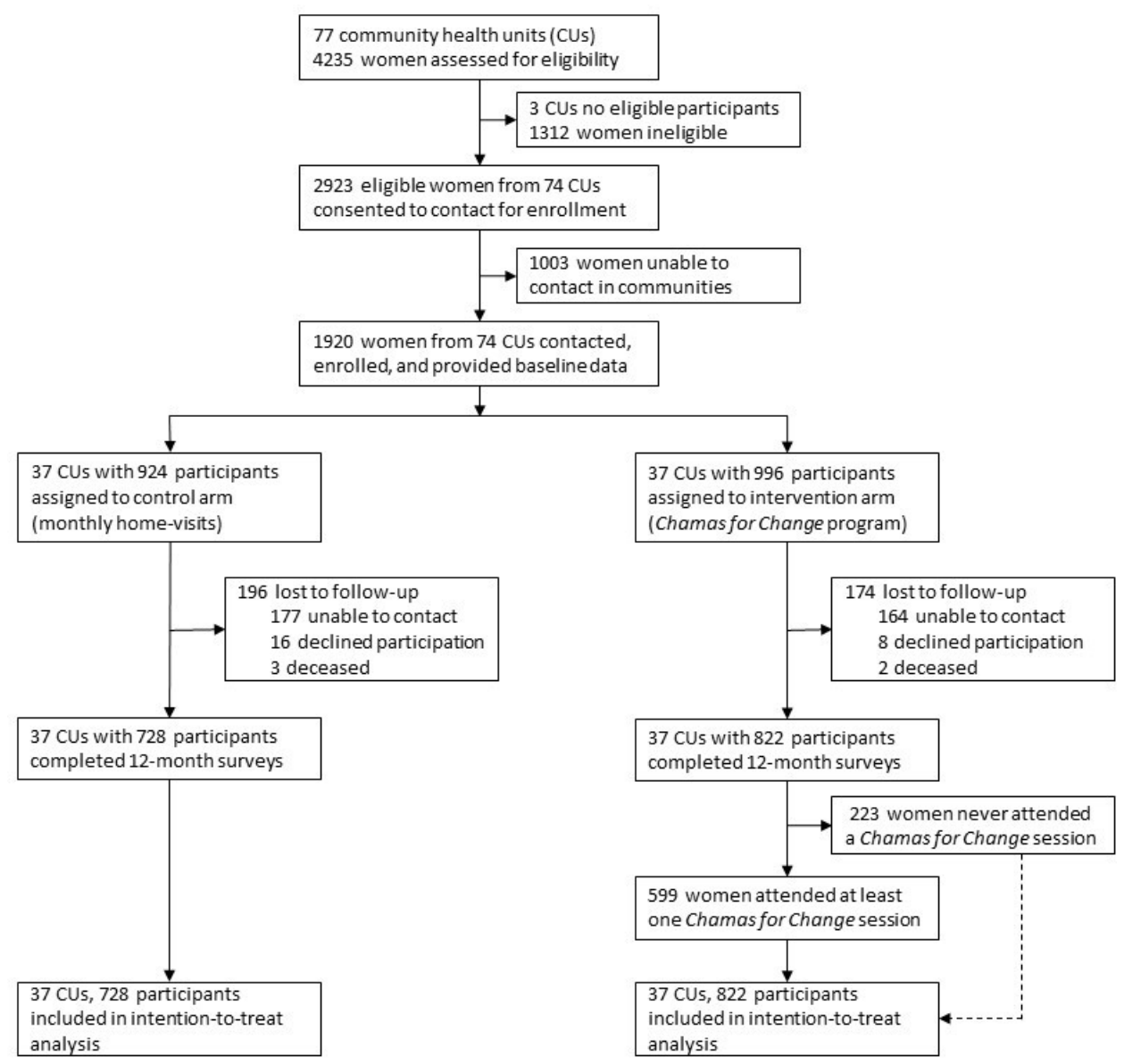

Figure 2 Trial profile.

associated with reaching a health facility (eg, too far, poor road conditions) $(32.1 \%)$ and medical emergencies (eg, abrupt labour with not enough time to travel) (11.5\%). In unadjusted models, we estimated a $7.4 \%$ (95\% CI 3.0 to 12.5) improvement in facility-based deliveries $(\mathrm{OR}=1.58$, $95 \%$ CI 0.97 to $2.55, \mathrm{p}=0.057$ ). Following adjustment and adjustment with imputation, this improvement was slightly attenuated to $6.4 \%$ (95\% CI 2.0 to 10.4 ) and $7.1 \%$ (95\% CI 3.0 to 11.4 ), respectively adjusted OR $((\mathrm{aOR}) 1=1.5995 \%$ CI 1.02 to $2.47, \mathrm{p}=0.042$; $\mathrm{aOR} 2=1.62$ $95 \%$ CI 1.06 to $2.49, \mathrm{p}=0.004$ ) (online supplemental table S3). Further, a sensitivity analysis restricting the intervention sample to women who attended Chamas at least once attenuated improvement in facility-based delivery by $5.2 \%(95 \%$ CI 1.5 to 9.5$)(\mathrm{OR}=1.4395 \%$ CI 0.92 to 2.24 , $\mathrm{p}=0.11$ ) (online supplemental file 4online supplemental file 4). We observed a relatively large amount of cluster heterogeneity as indicated by an ICC of 0.18 (figure 3 ).

We examined the effect of Chamas participation on secondary MNCH outcomes associated with demonstrated reductions in maternal and infant morbidity and mortality. Women in Chamas clusters improved in 48-hours postpartum visits (RD 15.3\%, 95\% CI 12.0 to 19.6), exclusive breastfeeding (RD 11.9\%, 95\% CI 7.2 to 16.9 ) and contraceptive adoption (RD $7.2 \%, 95 \%$ CI 2.6 to 12.9) compared with controls (table 2). Though not statistically significant, the RDs in achieving adequate ANC and adopting a long-acting method of contraception (ie, intrauterine device or implant) were also greater among Chamas participants. Restricting our intervention sample to women who attended Chamas at least once accentuated improvements in 48 hours postpartum visits adjusted RD ( (aRD) 19.6\%, 95\% CI 14.4 to 25.0 ) and exclusive breastfeeding (aRD $13.6 \%, 95 \%$ CI 7.8 to 19.8 ); conversely, we observed an attenuated effect with this restriction on contraceptive adoption (aRD 5.7\%, 95\% CI 0.7 to 11.1) (online supplemental table S4). Other sensitivity analyses did not meaningfully change results (online supplemental table S4).

We additionally assessed infant immunisation outcomes among live infants at follow-up. Infants born to women in Chamas demonstrated significant improvements in receiving the measles I vaccine by 12 months of age (RD 13.2\%, 95\% CI 9.1 to 18.4) and completing the recommended infant immunisation series per WHO (RD $15.6 \%, 95 \%$ CI 11.5 to 20.9) and Republic of Kenya $\mathrm{MOH}$ (RD $15.1 \%$, 95\% CI 10.4 to 20.3) guidelines (table 3). These results were unchanged after adjusting for covariates (online supplemental table S4). We estimated an unmeasured confounder (due to selection bias in those that reported the outcome) associated with both increased rate of vaccination and enrolment in intervention trial arm (compared with control) by $30 \%$ would 
Table 1 Baseline characteristics for intention-to-treat population (74 clusters, $n=1550$ )

\begin{tabular}{rrr}
$\begin{array}{r}\text { Control } \\
(\mathbf{N}=\mathbf{7 2 8})\end{array}$ & $\begin{array}{l}\text { Intervention } \\
(\mathbf{N}=\mathbf{8 2 2})\end{array}$ & $\begin{array}{l}\text { Overall } \\
\mathbf{( N = 1 5 5 0}\end{array}$ \\
\hline 37 & 37 & 74 \\
198288 & 226930 & 45218 \\
45433 & 47279 & 92712
\end{tabular}

$\mathrm{N}$ clusters

Total population

\begin{tabular}{lccc|}
\hline Rural & 33 & 32 & 65 \\
\hline Peri-urban & 3 & 3 & 6 \\
\hline Urban & 1 & 2 & 3 \\
\hline CHV experience (years) & $11.73(6.78)$ & $11.67(6.13)$ & $11.69(6.32)$ \\
\hline Maternal age & $26.63(6.21)$ & $27.10(6.55)$ & $26.88(6.40)$ \\
\hline $\begin{array}{l}\text { Gestational age (weeks) at enrolment, } \\
\text { median (IQR) }\end{array}$ & $22(18,25)$ & $22(18,25)$ & $22(18,25)$ \\
\hline Marital status & & & \\
\hline Divorced/separation & $11(1.5 \%)$ & $17(2.1 \%)$ & $28(1.8 \%)$ \\
\hline Married & $606(83.2 \%)$ & $686(83.5 \%)$ & $1292(83.4 \%)$ \\
\hline Single & $109(15.0 \%)$ & $115(14.0 \%)$ & $224(14.5 \%)$ \\
\hline Widowed & $2(0.3 \%)$ & $4(0.5 \%)$ & $6(0.4 \%)$ \\
\hline Maternal education & & & $137(8.8 \%)$ \\
\hline College or higher & $91(12.5 \%)$ & $46(5.6 \%)$ & $461(29.7 \%)$ \\
\hline Secondary or postprimary & $211(29.0 \%)$ & $250(30.4 \%)$ & $733(47.3 \%)$ \\
\hline Primary & $313(43.0 \%)$ & $420(51.1 \%)$ & $215(13.9 \%)$ \\
\hline Preprimary or none & $113(15.5 \%)$ & $102(12.4 \%)$ & $4(0.3 \%)$ \\
\hline Missing & $0(0.0 \%)$ & $4(0.5 \%)$ & $97(6.3 \%)$ \\
\hline Occupation & & & $32(2.1 \%)$ \\
\hline Contract/temporary worker & $49(6.7 \%)$ & $48(5.8 \%)$ & $448(28.9 \%)$ \\
\hline Permanently employed & $22(3.0 \%)$ & $10(1.2 \%)$ & $972(62.7 \%)$ \\
\hline Self-employed & $201(27.6 \%)$ & $247(30.0 \%)$ & $1(0.1 \%)$ \\
\hline Unemployed & $456(62.6 \%)$ & $516(62.8 \%)$ & $1(0.1 \%)$ \\
\hline Missing & $0(0.0 \%)$ & & \\
\hline
\end{tabular}

32

65

Health insurance coverage at time of delivery

\begin{tabular}{llcc}
\hline Yes & $412(56.6 \%)$ & $519(63.1 \%)$ & $931(60.1 \%)$ \\
\hline No & $285(39.1 \%)$ & $285(34.7 \%)$ & $570(36.8 \%)$ \\
\hline Missing & $31(4.3 \%)$ & $18(2.2 \%)$ & $49(3.2 \%)$ \\
\hline $\begin{array}{l}\text { Poverty probability index score* } \\
\text { \% poverty likelihood at national } \\
\text { poverty line }\end{array}$ & $56.79(20.69)$ & $53.61(19.45)$ & $55.13(20.11)$ \\
$\begin{array}{l}\text { Subcounty } \\
\text { Cherangany }\end{array}$ & $22.6 \%$ & $25.7 \%$ & $24.6 \%$ \\
\hline Kiminini & $229(31.5 \%)$ & & \\
\hline Kwanza & $145(19.9 \%)$ & $211(25.6 \%)$ & $440(28.4 \%)$ \\
\hline $\begin{array}{l}\text { Saboti } \\
\text { Previously pregnant }\end{array}$ & $193(26.5 \%)$ & $216(26.9 \%)$ & $317(20.5 \%)$ \\
\hline Parity & $161(22.1 \%)$ & $223(27.1 \%)$ & $384(24.8 \%)$ \\
\hline Previous modern contraceptive use & $584(80.2 \%)$ & $623(75.8 \%)$ & $1207(77.8 \%)$ \\
\hline Yes & $2.29(1.62)$ & $2.58(1.57)$ & $2.35(1.56)$ \\
\hline
\end{tabular}


Table 1 Continued

\begin{tabular}{|c|c|c|c|}
\hline & $\begin{array}{l}\text { Control } \\
(\mathrm{N}=728)\end{array}$ & $\begin{array}{l}\text { Intervention } \\
(\mathrm{N}=822)\end{array}$ & $\begin{array}{l}\text { Overall } \\
(\mathrm{N}=1550)\end{array}$ \\
\hline No & $214(36.6 \%)$ & $210(33.7 \%)$ & $424(35.1 \%)$ \\
\hline Missing & $48(8.2 \%)$ & $32(5.1 \%)$ & $80(6.6 \%)$ \\
\hline \multicolumn{4}{|l|}{ Previous facility delivery } \\
\hline Yes & $228(39.0 \%)$ & $279(44.8 \%)$ & $507(42.0 \%)$ \\
\hline No & $162(27.7 \%)$ & $161(25.8 \%)$ & $323(26.8 \%)$ \\
\hline Missing & $194(33.2 \%)$ & $183(29.4 \%)$ & $377(31.2 \%)$ \\
\hline \multicolumn{4}{|c|}{ Total ANC visits in previous pregnancy } \\
\hline 0 & $6(1.0 \%)$ & $21(3.4 \%)$ & $27(2.2 \%)$ \\
\hline 1 & $18(3.1 \%)$ & $22(3.5 \%)$ & $40(3.3 \%)$ \\
\hline 2 & $29(5.0 \%)$ & $39(6.3 \%)$ & $68(5.6 \%)$ \\
\hline 3 & 119 (20.4\%) & $128(20.5 \%)$ & 247 (20.5\%) \\
\hline 4 & $135(23.1 \%)$ & $172(27.6 \%)$ & $307(25.4 \%)$ \\
\hline$>4$ & $74(12.7 \%)$ & $54(8.7 \%)$ & $128(10.6 \%)$ \\
\hline Missing & $203(34.8 \%)$ & $187(30.0 \%)$ & $390(32.3 \%)$ \\
\hline \multicolumn{4}{|l|}{ Previoust } \\
\hline Miscarriage & $23(3.9 \%)$ & $26(4.2 \%)$ & $49(4.1 \%)$ \\
\hline Stillbirth & $9(1.5 \%)$ & $16(2.6 \%)$ & $25(2.1 \%)$ \\
\hline Neonatal death & $8(1.4 \%)$ & $5(0.8 \%)$ & $13(1.1 \%)$ \\
\hline Infant death & $8(1.4 \%)$ & $5(0.8 \%)$ & $13(1.1 \%)$ \\
\hline Child death under 5 & $6(1.0 \%)$ & 2 (0.3\%) & 8 (0.7\%) \\
\hline Child death over 5 & $3(0.5 \%)$ & $2(0.3 \%)$ & $5(0.4 \%)$ \\
\hline
\end{tabular}

*Scores and \% poverty likelihood calculated using validated 2015 Kenya Poverty Probability Index.

†Miscarriage (up to 28 weeks gestation); stillbirth (after 28 weeks gestation); neonatal death (0-28 days old); infant death (1-12 months old); child death (1-5 years old).

$\mathrm{ANC}$, antenatal care; $\mathrm{CHV}$, community health volunteer.

be required to explain away these observed significant differences.

Maternal and infant mortality and morbidity outcomes are presented in table 4 with no significant differences between trial arms; however, the trial was not powered to detect differences in these relatively rare outcomes. Overall, we observed a protective effect of Chamas participation against maternal $(\mathrm{RD}-4.7 \%, 95 \% \mathrm{CI}-9.4$ to $0.1)$ and infant $(\mathrm{RD}-3.9 \%, 95 \% \mathrm{CI}-8.6$ to 0.3$)$ morbidity. We recorded five participant mortalities during the trial (two in intervention and three in control). Three deaths were attributed to maternal causes of mortality, notably: one due to obstructed labour, one due to postcaesarian infection and one due to eclampsia; the remaining

Table 2 Primary and secondary outcomes: facility-based delivery, care seeking and vaccination

\begin{tabular}{|c|c|c|c|c|c|}
\hline & Controlł & Intervention $¥$ & Risk difference $(95 \% \mathrm{Cl})$ & Odds ratio $(95 \% \mathrm{Cl})$ & P value* \\
\hline Facility-based delivery & $514(73.0 \%)$ & $653(80.9 \%)$ & $7.4 \%$ (3.0\% to $12.5 \%)$ & 1.58 (0.969 to 2.55$)$ & 0.057 \\
\hline Adequate ANC care $\dagger$ & $507(69.6 \%)$ & 587 (71.4\%) & $3.2 \%(-1.5 \%$ to $7.7 \%)$ & 1.18 (0.82 to 1.68$)$ & 0.375 \\
\hline Postnatal CHV visit & $97(13.6 \%)$ & $241(30.1 \%)$ & $15.3 \%(12.0 \%$ to $19.6 \%$ & 3.22 (1.50 to 6.93 ) & 0.003 \\
\hline $\begin{array}{l}\text { Exclusive breast feeding for } \\
6 \text { months }\end{array}$ & $383(56.7 \%)$ & $521(67.2 \%)$ & $11.9 \%(7.2 \%$ to $16.9 \%)$ & 1.77 (1.12 to 2.80$)$ & 0.014 \\
\hline Contraceptive use & $472(65.5 \%)$ & $581(71.8 \%)$ & $7.2 \%(2.6 \%$ to $12.9 \%)$ & $1.41(1.03$ to 1.93$)$ & 0.034 \\
\hline $\begin{array}{l}\text { Long-acting reversible } \\
\text { contraceptive use }\end{array}$ & $242(51.3 \%)$ & $326(56.1 \%)$ & $7.1 \%(0.9 \%$ to $13.3 \%)$ & 1.34 (0.95 to 1.91$)$ & 0.099 \\
\hline
\end{tabular}

${ }^{*} \mathrm{P}$ value is for $\mathrm{OR}$ from mixed effect logistic regression.

†Adequate ANC care is defined as attending at least four ANC visits per Republic of Kenya Ministry of Health guidelines.

fDenominators are based on number of women reporting the particular outcome. See Online supplemental table S1 for details.

$\mathrm{ANC}$, antenatal care; $\mathrm{CHV}$, community health volunteer. 


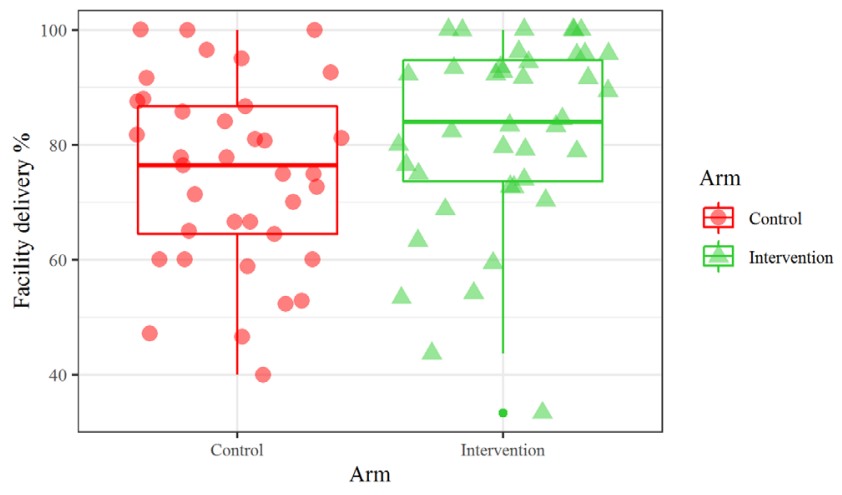

Figure 3 Cluster outcome rates.

two deaths were attributed to complications of cervical cancer. None of these mortalities was directly associated with trial participation. Across both trial arms, we recorded 43 perinatal deaths (ie, deaths during the first week of life), 15 neonatal deaths and 25 infant deaths.

\section{DISCUSSION}

In Kenya and other resource-limited settings, effective community-based strategies are increasingly needed to reduce maternal and infant deaths. Encouraging facilitybased delivery is one well-known and highly effective strategy to achieve this goal. ${ }^{29}$ Despite government-led initiatives that support access to health services-such as the CHS and elimination of delivery-related fees at public facilities announced in 2013-facility-based deliveries nationally $(61.2 \%)$ and in our study area $(56.5 \%)$ are still lower than needed to sufficiently reduce the MMR and IMR. ${ }^{130}$ Against this backdrop of underused intrapartum services, we rigorously tested a communitybased women's health education programme designed to improve facility-based deliveries and other $\mathrm{MNCH}$ practices. We evaluated outcomes in an ITT sample derived from a geographically diverse catchment area. These analyses produced findings that mimic real-world scenarios in which perfect programme attendance is unlikely. We found that facility-based delivery and other key MNCH practices significantly improved in the intervention arm, supporting our hypothesis.

During the past decade, community health workers have emerged as a focal point of global discussions about advancing primary healthcare systems. ${ }^{6}$ There is substantial evidence to support the integration of these workers in the delivery of preventive $\mathrm{MNCH}$ interventions including, but not limited to: malaria prevention, health education, breastfeeding promotion, essential newborn care and psychosocial support. ${ }^{7}$ Leveraging a well-trained, community-based, health worker corps to mobilise preventive health measures has demonstrated promising reductions in maternal and neonatal mortality, particularly in LMIC contexts; however, much of the existing literature focuses on door-to-door as opposed to group-based delivery models. ${ }^{8}$

Chamas expands on this existing community infrastructure to attempt to reach some of the most vulnerable members of Kenyan society-pregnant and postpartum women in poor, rural communities. The CHS provided an established workforce of trained CHVs who-with bolstered support, supervision and mechanisms to increase work efficiency-significantly improved $\mathrm{MNCH}$ outcomes. Notably, CHVs facilitating Chamas spent fewer volunteer hours than counterparts performing door-todoor visits (3 vs 7.5 hours); moreover, women participating in Chamas achieved a greater number of total visits (12 vs 9 CHV contacts) than those visited at home. Several CHV-related barriers to effective implementation of the current CHS model have been identified, including: the absence of consistent supervision, inadequate health training, poor linkages to health facilities, lack of accountability and absence of remunerative pay. ${ }^{31}$ Our data support that by providing CHVs with additional oversight, a structured curriculum and an opportunity to economise their time through a group-based delivery model, the Chamas programme helped narrow the margin between aspirational and achievable $\mathrm{MNCH}$ improvements.

Moreover, we recognise that opportunities for CHV financial remuneration are critical to ensuring the

Table 3 Infant immunisation outcomes

\begin{tabular}{|c|c|c|c|c|c|}
\hline & Control† & Intervention† & Risk difference $(95 \% \mathrm{Cl})$ & Odds ratio $(95 \% \mathrm{Cl})$ & P value* \\
\hline $\begin{array}{l}\text { Infants who received OPV } 0 \text { within } \\
2 \text { weeks of birth }\end{array}$ & $341(64.6 \%)$ & $361(66.0 \%)$ & $1.7 \%(-3.6 \%$ to $8.4 \%)$ & $1.08(0.77$ to 1.51$)$ & 0.663 \\
\hline $\begin{array}{l}\text { Infants who received measles I by } \\
12 \text { months of age }\end{array}$ & $328(74.0 \%)$ & $339(87.6 \%)$ & $13.2 \%(9.1 \%$ to $18.4 \%)$ & 2.71 (1.45 to 5.04$)$ & 0.002 \\
\hline $\begin{array}{l}\text { Fully immunised infants ( } \leq 12 \\
\text { months) per WHO standards }\end{array}$ & $324(73.6 \%)$ & $352(88.9 \%)$ & $15.6 \%(11.5 \%$ to $20.9 \%)$ & 3.52 (1.74 to 7.12 ) & $<0.001$ \\
\hline $\begin{array}{l}\text { Fully-immunised infants ( } \leq 12 \\
\text { months) per Republic of Kenya } \\
\text { MOH standards }\end{array}$ & $320(73.1 \%)$ & $348(87.7 \%)$ & $15.1 \%(10.4 \%$ to $20.3 \%)$ & 3.16 (1.61 to 6.21$)$ & $<0.001$ \\
\hline
\end{tabular}

${ }^{*} \mathrm{P}$ value is for $\mathrm{OR}$ from mixed effect logistic regression.

†Denominators are based on number of women reporting the particular outcome. See online supplemental table S1 for details.

OPV, oral polio vaccine. 
Table 4 Maternal and infant mortality and morbidity outcomes

\begin{tabular}{|c|c|c|c|c|c|}
\hline & Control & Intervention & Risk difference $(95 \% \mathrm{Cl})$ & OR $(95 \% \mathrm{Cl})$ & P value* \\
\hline Maternal mortality & $3(<0.1 \%)$ & $2(<0.1 \%)$ & - & - & - \\
\hline Maternal morbidity† & $136(18.7 \%)$ & $110(13.4 \%)$ & $-4.7 \%(-9.4 \%$ to $0.1 \%)$ & $0.68(0.42$ to 1.10$)$ & 0.118 \\
\hline Miscarriage & $16(2.2 \%)$ & $13(1.6 \%)$ & $-0.2 \%(-1.3 \%$ to $0.8 \%)$ & $0.85(0.30$ to 2.38$)$ & 0.751 \\
\hline Stillbirth & $16(2.2 \%)$ & $12(1.5 \%)$ & $-0.6 \%(-1.7$ to $0.3 \%)$ & 0.64 (0.27 to 1.56$)$ & 0.331 \\
\hline Perinatal death $\ddagger$ & $22(3.1 \%)$ & $21(2.6 \%)$ & $-0.5 \%(-1.9 \%$ to $0.8 \%)$ & $0.83(0.42$ to 1.66$)$ & 0.601 \\
\hline Neonatal death & $6(0.87 \%)$ & $9(1.13 \%)$ & $0.2 \%(-0.6 \%$ to $0.7 \%)$ & 1.29 (0.39 to 4.29$)$ & 0.674 \\
\hline Infant death & $13(1.9 \%)$ & $12(1.5 \%)$ & $-0.2 \%(-1.3 \%$ to $0.8 \%)$ & 0.83 (0.27 to 2.5$)$ & 0.689 \\
\hline Low birth weight & $118(16.0 \%)$ & $157(18.9 \%)$ & $1.9 \%(-1.6 \%$ to $5.6 \%)$ & $1.16(0.70$ to 1.90$)$ & 0.570 \\
\hline Infant morbidity§ & $132(18.68 \%)$ & $118(16.74 \%)$ & $-3.9 \%(-8.6 \%$ to $-0.3 \%)$ & $0.76(0.51$ to 1.15$)$ & 0.194 \\
\hline
\end{tabular}

${ }^{*} \mathrm{P}$ value is for $\mathrm{OR}$ from mixed effect logistic regression.

†Maternal morbidity defined as any health condition attributed to and/or aggravated by pregnancy and childbirth that has a negative impact on the woman's well-being, including the following complications: miscarriage (<28 weeks), stillbirth ( $>28$ weeks), gestational diabetes, preeclampsia, eclampsia, postpartum infection, postpartum haemorrhage, or obstructed labour.

¥Perinatal deaths (first week of life), neonatal deaths (through 28th day of life), infant deaths (through first year of life).

§Infant morbidity defined as any health condition that affects mortality rate during the first-year of life including low birth weight $(<2.5 \mathrm{~kg})$, perinatal disorders (gestational diabetes, preeclampsia, eclampsia), infant immunisation adherence, exclusive breastfeeding, and deliveryrelated complications (obstructed labour, neonatal resuscitation).

sustainability of this programme and plan to prioritise this in future attempts to scale. Globally, several national governments-including Brazil, Pakistan, Ethiopia and India-have made concerted efforts to move away from the traditional $\mathrm{CHV}$ role as an unpaid, lightly trained member of the community and instead towards creating a highly skilled, compensated health worker capable of providing treatments and implementing preventive health measures. ${ }^{32}$ We anticipate that financial support-in conjunction with advancements in training and ongoing community involvement-will catalyse even greater success for the Chamas programme. We plan to achieve this vision by continuing our close collaboration with the Republic of Kenya MOH to advocate for increased allocation of local and national funds to bolster community health infrastructure as well as by encouraging $\mathrm{MOH}$ representatives to integrate Chamas within existing health strategies.

Our intervention approach-group-based women's health education delivered by CHVs during pregnancy and postpartum-champions a theory of change that prioritises three key areas: (1) empowering women with health and social literacy, (2) establishing a network of supportive peers and (3) providing women with an opportunity to gain financial capital (GISHE). This third component is distinct from preceding strategies that promote a group-based, lay health worker-led model for $\mathrm{MNCH} .{ }^{12}$ We suspect this multipronged approach that prioritises these three critical components, in addition to leveraging an established CHV workforce, plays a significant role in enhancing positive outcomes. Evidence suggests peer support and peer accountability may enhance the likelihood of practicing positive health behaviours. ${ }^{14}$ Further, there is a growing body of evidence that suggests coupling health education with microfinance may improve women's health; however, most literature on associated reproductive health outcomes focus on contraceptive uptake and adherence to HIV/AIDS treatment. ${ }^{33}$ Among group members who participated in GISHE, we speculate the opportunity to generate savings likely served a dual purpose of motivating Chamas attendance while helping some participants overcome financial barriers to accessing care. Future analyses will attempt to dissect the influence of each of these components on overall programme success. Finally, while most programmes intervene during distinct time periods (eg, prenatal, intrapartum), Chamas embraces a life-course approach by engaging women throughout the first 1000 days of their child's life. We anticipate families who continue in Chamas likely experience health and social benefits throughout subsequent years. These effects are largely unexplored and will be the focus of future trials.

Preceding trials have examined the effect of similar community-based women's health education groups on key MNCH behaviours, including facility-based delivery. ${ }^{12}$ A meta-analysis combining results from seven cluster randomised controlled trials conducted in resourcelimited settings (ie, Nepal, Bangladesh, Malawi and India) found no evidence of intervention effects on facilitybased delivery (OR $1.02,95 \%$ CI 0.93 to $1.12 ; \mathrm{I}^{2}=21.4 \%$, $95 \%$ CI 0 to $65.8 \%$ ); similarly, these analyses revealed no evidence of effect on uptake of ANC or exclusive breastfeeding. ${ }^{12}$ Although data comparability is limited by differences in trial design, setting and programme structure (ie, absence of table banking), we observed significantly higher odds of facility-based delivery and exclusive breastfeeding in the intervention arm. Our findings strengthen evidence from an earlier Chamas pilot that similarly demonstrated increased odds of achieving these outcomes compared with the standard of care in rural 
western Kenya. ${ }^{15}$ Collectively, these findings highlight our intervention's potential to improve MNCH outcomes by leveraging existing community health resources and infrastructure in settings like Kenya.

This work has several limitations. First, by focusing our recruitment effort at the facility level, we limited our sample to women attending ANC visits. Though the majority $(96.1 \%)$ of Kenyan women attend at least one ANC visit during their pregnancy, this estimate is likely lower among rural communities with poorer access to care. ${ }^{1}$ Future studies will ideally combine both facility and community-based recruitment strategies to foster a more inclusive cohort. Second, we experienced significant recruitment challenges that substantially reduced our sample size. Processes to contact eligible participants outside of health facilities proved arduous and complicated as locator data (eg, home address, phone number) were often unreliable. This loss to follow-up between health facilities and the community likely introduced selection bias as we suspect women were not missing at random. It is possible these missing data reflect an inability to pay for cell phones (or data) or perhaps limited access in rural, hard-to-reach communities; thus, we may have failed to enrol some of the most socioeconomically disadvantaged members of these communities. These challenges highlight a need to not only improve our strategy but also to strengthen continuity to ensure vulnerable members of society are accounted for. Third, large amounts of missing data compromised the interpretability of certain outcomes, most notably infant immunisations. Despite established processes to monitor data quality, data collectors reported several challenges that compromised questionnaire completion. Obstacles included interruptions due to competing obligations (ie, child care) and lack of a private interview setting. Further, relatively few participants possessed $\mathrm{MOH}$ Maternal Child Health booklets and among those who had them, few recorded data. This limitation may have introduced selection bias as mothers with completed records may have had greater access to or higher quality care. Alternatively, this could also reflect limited booklet availability, poor record-keeping or other structural limitations worthy of consideration. Fourth, we examined our primary outcome (facility-based delivery) as an aggregate measure, which limited our ability to discern where improvement occurred in the health system (ie, public vs private health sector). Future studies will collect detailed measures to clarify these data and guide targeted approaches to improving the health system at large. Finally, we observed a large amount of cluster heterogeneity indicating significant though anticipated community-level variation. Compositional effects within or between clusters such as proximity to health facilities, availability of service providers and fidelity of programme implementation may contribute to this variation. These effects may partly explain the unexpected attenuated difference in facility-based delivery in our sensitivity analysis of Chamas attendees. Clarifying community-level factors contributing to variable outcomes may help bridge these observed geographic disparities.

These limitations are balanced by several noteworthy strengths of our study. We detected significant results in our primary and secondary outcomes using an intention to treat approach. These observed effects were generally robust-for example, not meaningfully changed following adjustment or imputation in our sensitivity analyses. The cluster randomised controlled design, implemented in a large and geographically diverse population, enhances generalisability of these findings. We saw no contamination across trial arms and minimised potential for information bias by masking data collectors, investigators and analysts to cluster allocation throughout the trial. Further, by imposing relatively few exclusion criteria and a generous gestational age cut-off, we attempted to broaden inclusion to women who may have sought late ANC due to structural (eg, distance to facility) or behavioural (eg, delayed awareness of pregnancy) factors. Finally, it is worth noting that the proportion of facility-based deliveries, among other outcomes, was higher in both trial arms (80.9\% intervention, $73.0 \%$ control) relative to county-level $(56.5 \%)$ and national $(61.2 \%)$ estimates. ${ }^{1}$ It is possible study procedures—such as training, supervision or general awareness of the trialled to CHVs in control CUs being more likely to deliver standard of care, which might explain these observations.

Chamas offers an innovative approach to improve $\mathrm{MNCH}$ in resource-limited settings with significant health policy implications. This intervention demonstrated significant improvements in $\mathrm{MNCH}$ outcomes relative to the current standard of care; policymakers should take note of this strategy as they attempt to improve current initiatives. Since the programme's inception, we have emphasised the importance of collaboration with and investment from key stakeholders, including but not limited to: women, community leaders, CHVs and $\mathrm{MOH}$ representatives at the county and national level. We respond to qualitative feedback from these stakeholders to ensure the programme iteratively responds to the needs of its beneficiaries and remains community driven. These commitments to collaboration and feedback inspire confidence in our programme's continued success. As we move towards scaling and integrating Chamas, our next steps will focus on addressing costeffectiveness and enhancing adaptability to new settings.

Finally, though these results highlight Chamas' potential to improve uptake of life-saving MNCH interventions, we must acknowledge the importance of promoting both access to as well as delivery of high-quality care to achieve sustainable health outcome improvement. As the global community works towards integrating more effective and efficient mechanisms for improving health for our most vulnerable populations, it is critical to simultaneously bolster the quality of services provided. Motivating communities to practice behaviours will only prove successful if health facilities and local governments maintain a similar commitment towards providing 
adequate staffing, maintaining supplies and promoting a welcoming environment for women. Our hope is that Chamas and other community-based programmes will support communities in demanding higher quality care and hold those in power accountable to strengthening systems for all women and children.

In summary, Chamas participation significantly improved MNCH outcomes compared with the standard of care in western Kenya. This trial contributes robust data from sub-Saharan Africa that strengthens evidence to support community-based, women's health education groups for $\mathrm{MNCH}$ in resource-limited settings.

\section{Author affiliations}

${ }^{1}$ Medicine and Pediatrics, Massachusetts General Hospital, Boston, Massachusetts, USA

${ }^{2}$ Population Health, Academic Model Providing Access to Healthcare, Eldoret, Kenya ${ }^{3}$ Obstetrics and Gynecology, The University of British Columbia, Vancouver, British Columbia, Canada

${ }^{4}$ Health Systems Design and Global Health, Icahn School of Medicine at Mount Sinai, New York City, New York, USA

${ }^{5}$ Child Health and Paediatrics, Moi University College of Health Sciences, Eldoret, Kenya

${ }^{6}$ Obstetrics and Gynecology, University of Toronto, Toronto, Ontario, Canada

${ }^{7}$ Medicine, Indiana University School of Medicine, Indianapolis, Indiana, USA

Acknowledgements We thank our study participants, community health volunteers, research assistants, and staff without whom this work would not be possible. We additionally thank the subcounty and county $\mathrm{MOH}$ representatives in Trans Nzoia for their support and collaboration. We are grateful for the mentorship and thoughtful feedback provided by our colleagues: Dr Donald C. Cole (Dalla Lana School of Public Health, University of Toronto), Dr K.S. Joseph (Department of Obstetrics and Gynecology, School of Population and Public Health, University of British Columbia), and Dr. Wendy Prudhomme 0'Meara (Duke Global Health Institute, Duke University). This trial was made possible through the generous support of the Saving Lives at Birth (SL@B) partnership. The authors alone are responsible for the views expressed in this article and they do not necessarily represent the views, decisions, or policies of the SL@B partners or the institutions with which they are affiliated.

Contributors AC-D, LJR and JJS conceptualised, sought and obtained funding for this study. LYM and MLS drafted the study protocol, developed data collection tools and oversaw all data management processes led by GA. JB and LYM developed the statistical analysis plan, with critical feedback provided by all coauthors. AJ, JEl and SC oversaw all research activities and coordinated research staff throughout the trial. JB conducted all statistical analyses with significant input from LYM. All authors assisted in interpreting results. LYM and JB authored the first draft of this article. All authors contributed to reviewing and editing the final draft of this article for intellectual content. All authors approved submission of this manuscript for publication.

Funding This study was supported by Grand Challenges Canada (0755-03).

Map disclaimer The depiction of boundaries on this map does not imply the expression of any opinion whatsoever on the part of BMJ (or any member of its group) concerning the legal status of any country, territory, jurisdiction or area or of its authorities. This map is provided without any warranty of any kind, either express or implied.

Competing interests None declared.

Patient consent for publication Not required.

Ethics approval We received ethics approvals from the Institutional Research Ethics Committee at Moi University and Moi Teaching and Referral Hospital (IREC/2018/269) and Institutional Review Board at Indiana University (1905296355).

Provenance and peer review Not commissioned; externally peer reviewed.

Data availability statement The de-identified data set and a data dictionary will be made available upon reasonable request with publication of the trial. Inquiries can be made to the corresponding author of this manuscript (Dr Lauren Y. Maldonado, lymaldonado@mgh.harvard.edu).
Supplemental material This content has been supplied by the author(s). It has not been vetted by BMJ Publishing Group Limited (BMJ) and may not have been peer-reviewed. Any opinions or recommendations discussed are solely those of the author(s) and are not endorsed by BMJ. BMJ disclaims all liability and responsibility arising from any reliance placed on the content. Where the content includes any translated material, BMJ does not warrant the accuracy and reliability of the translations (including but not limited to local regulations, clinical guidelines, terminology, drug names and drug dosages), and is not responsible for any error and/or omissions arising from translation and adaptation or otherwise.

Open access This is an open access article distributed in accordance with the Creative Commons Attribution Non Commercial (CC BY-NC 4.0) license, which permits others to distribute, remix, adapt, build upon this work non-commercially, and license their derivative works on different terms, provided the original work is properly cited, appropriate credit is given, any changes made indicated, and the use is non-commercial. See: http://creativecommons.org/licenses/by-nc/4.0/.

\section{ORCID iDs}

Lauren Y Maldonado http://orcid.org/0000-0002-8352-0815

Jeffrey Bone http://orcid.org/0000-0001-7704-1677

\section{REFERENCES}

1 Kenya National Bureau of Statistics. 2014 Kenya demographic and health survey (KDHS). Nairobi, Kenya Demographic Health Survey; 2015.

2 UNICEF. Kenya: demographics, health, and infant mortality. Available: https://data.unicef.org/country/ken/ [Accessed 8 Mar 2020].

3 Graham WJ, Fitzmaurice AE, Bell JS, et al. The familial technique for linking maternal death with poverty. Lancet 2004;363:23-7.

4 Mills A. Health care systems in low- and middle-income countries. $N$ Engl J Med 2014;370:552-7.

5 Bhutta ZA, Black RE. Global maternal, newborn, and child health-so near and yet so far. N Engl J Med 2013;369:2226-35.

6 Singh P, Sachs JD. 1 million community health workers in subSaharan Africa by 2015. Lancet 2013;382:363-5.

7 Gilmore B, McAuliffe E. Effectiveness of community health workers delivering preventive interventions for maternal and child health in low- and middle-income countries: a systematic review. BMC Public Health 2013;13:847.

8 Lewin S, Munabi-Babigumira S, Glenton C, et al. Lay health workers in primary and community health care for maternal and child health and the management of infectious diseases. Cochrane Database Syst Rev 2010;3:CD004015.

9 Republic of Kenya Ministry of Health. Taking the Kenya essential package for health to the community: a strategy for the delivery of level one services (community health strategy). Nairobi, Kenya Ministry of Health; 2006.

10 Ministry of Health Division of Community Health Services. Community health volunteers (CHVs): basic modules Handbook. Nairobi, Kenya: USAID, 2013

11 World Health Organization. Optimizing health worker roles to improve access to key maternal and newborn health interventions through task shifting. Geneva, Switzerland; 2012.

12 Seward N, Neuman M, Colbourn T, et al. Effects of women's groups practising participatory learning and action on preventive and careseeking behaviours to reduce neonatal mortality: a meta-analysis of cluster-randomised trials. PLoS Med 2017;14:e1002467.

13 Lassi ZS, Bhutta ZA. Community-Based intervention packages for reducing maternal and neonatal morbidity and mortality and improving neonatal outcomes. Cochrane Database Syst Rev 2015;3:CD007754.

14 Dennis C-L. Peer support within a health care context: a concept analysis. Int J Nurs Stud 2003;40:321-32.

15 Maldonado LY, Songok JJ, Snelgrove JW, et al. Promoting positive maternal, newborn, and child health behaviors through a groupbased health education and microfinance program: a prospective matched cohort study in Western Kenya. BMC Pregnancy Childbirth 2020;20:288.

16 Khan KS, Wojdyla D, Say L, et al. Who analysis of causes of maternal death: a systematic review. Lancet 2006;367:1066-74.

17 Lawn JE, Cousens S, Zupan J. Lancet neonatal survival steering T. 4 million neonatal deaths: when? where? why? Lancet 2005;365:891-900.

18 Buor D, Bream K. An analysis of the determinants of maternal mortality in sub-Saharan Africa. J Womens Health 2004;13:926-38. 
19 Temmerman M, Khosla R, Bhutta ZA, et al. Towards a new global strategy for women's, children's and adolescents' health. BMJ 2015;351:h4414

20 Conde-Agudelo A, Rosas-Bermúdez A, Kafury-Goeta AC. Birth spacing and risk of adverse perinatal outcomes: a meta-analysis. JAMA 2006;295:1809-23.

21 Lund N, Andersen A, Hansen ASK, et al. The effect of oral polio vaccine at birth on infant mortality: a randomized trial. Clin Infect Dis 2015;61:1504-11.

22 Innovations for Poverty Action (IPA). Kenya 2015 PPI: Scorecards and Look-Up tables. New Haven, CT: Innovations for Poverty Action, 2018.

23 Rutterford C, Copas A, Eldridge S. Methods for sample size determination in cluster randomized trials. Int $J$ Epidemiol 2015;44:1051-67.

24 Quartagno MC J. jomo: a package for multilevel joint modelling multiple imputation. Available: https://cran.r-project.org/package $=$ jomo [Accessed 20 Feb 2020].

25 Rubin D. Multiple imputation for nonresponse in surveys. Wiley, 1987.

26 Tauil MdeC, Sato APS, Waldman EA. Factors associated with incomplete or delayed vaccination across countries: a systematic review. Vaccine 2016;34:2635-43.
27 VanderWeele TJ, Ding P. Sensitivity analysis in observational research: introducing the E-Value. Ann Intern Med 2017;167:268-74.

28 R Core Team. R: a language and environment for statistical computing. $\mathrm{R}$ foundation for statistical computing, 2019. Available: http://www.R-project.org/

29 Campbell OMR, Graham WJ, Lancet Maternal Survival Series steering group. Strategies for reducing maternal mortality: getting on with what works. Lancet 2006;368:1284-99.

30 Gitobu CM, Gichangi PB, Mwanda WO. The effect of Kenya's free maternal health care policy on the utilization of health facility delivery services and maternal and neonatal mortality in public health facilities. BMC Pregnancy Childbirth 2018;18:77.

31 McCollum R, Otiso L, Mireku M, et al. Exploring perceptions of community health policy in Kenya and identifying implications for policy change. Health Policy Plan 2016;31:10-20.

32 Haines A, Sanders D, Lehmann U, et al. Achieving child survival goals: potential contribution of community health workers. Lancet 2007;369:2121-31.

33 O'Malley TL, Burke JG. A systematic review of microfinance and women's health literature: directions for future research. Glob Public Health 2017;12:1433-60. 